\title{
EXTENDING THE RANGE OF A LOW ENERGY SR-SOURCE TO HARD X-RAYS *
}

\author{
M. Abo-Bakr, W. Anders, K. Bürkmann, V. Dürr, P. Kuske, R. Müller, K. Ott, M. Scheer, \\ E. Weihreter $^{\dagger}$, G. Wüstefeld, BESSY, Berlin, H. Winick, SSRL/SLAC, Stanford, \\ H. Kaiser, J. Koupsidis, M. Marx, G.-A. Voss, DESY, Hamburg
}

\begin{abstract}
An outline design is presented for a SR-source providing high brilliance VUV/soft X-Rays and high flux hard Xrays based on existing hardware of the BESSY I storage ring, which will be shut down by the end of 1999. Essential features are a 6-cell double bend achromat lattice, with low beta in three of the 6 long straight sections to facilitate the use of superconducting multipole wigglers. Beam optical implications are discussed together with technical modifications of existing hardware and possible operation at $1.0 \mathrm{GeV}$. A design of a $7.5 \mathrm{~T}$ multipole wiggler is given.
\end{abstract}

\section{INTRODUCTION}

The second generation SR-source BESSY I in Berlin, which consists of an $800 \mathrm{MeV}$ storage ring [1] with a full energy injector synchrotron [2], has been used over the last 18 years for a large scientific program in the VUV/soft Xray range. The recent start of user operation of the third generation ring BESSY II makes it necessary to close down BESSY I by the end of 1999. Based on the available hardware an outline design is presented for a SR-source providing high brilliance VUV/soft X-rays as well as high flux hard X-rays from insertion devices [3]. The increasing demand of hard X-rays for structural analysis, protein crystallography, environmental research and many other activities has motivated the present ring concept, which would allow to rejuvenate the old BESSY I ring making use of a large part of the subsystems and hardware components for a versatile SR-source covering the energy range from $10 \mathrm{eV}$ to about $20 \mathrm{keV}$.

There are two essential modifications of the BESSY I ring which will allow to achieve this important spectral extension: the installation of superconducting 7.5 T multipole wigglers and an upgrade of the beam energy to $1.0 \mathrm{GeV}$.

High field multipole wigglers are best arranged at places of low beta function values in both planes. This will provide for higher flux density of the photon beams, while at the same time keeping the undesirable linear and nonlinear focusing effects of the wiggler small. Further increase in photon energy is achieved by modifying the bending magnets to permit $1.0 \mathrm{GeV}$ operation.

Changing the original lattice of BESSY I with 4 straight

\footnotetext{
* Work supported by the Bundesministerium für Bildung, Wissenschaft, Forschung und Technologie and by the Land Berlin and the US Department of Energy.

† Email: weihreter@bessy.de
}

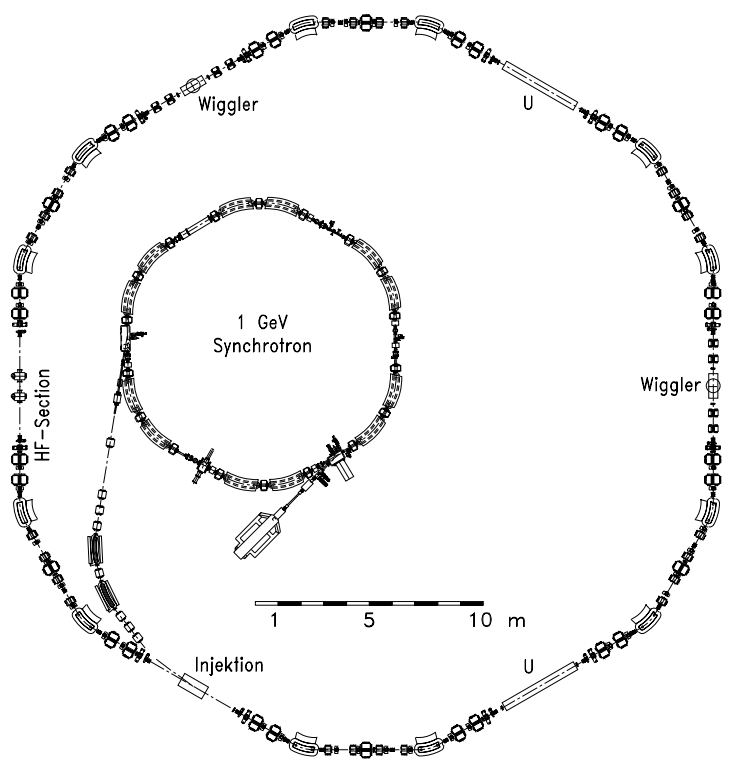

Figure 1: Accelerator and storage ring lay out.

sections of intermediate length $(3.6 \mathrm{~m})$ to one of 6 straight sections, each $6 \mathrm{~m}$ long, the facility can provide up to 20 dipole beamlines with a critical photon energy of $\epsilon_{c}=$ $1.25 \mathrm{keV}$, two straight sections for the installation of $7.5 \mathrm{~T}$ multipole wigglers $\left(\epsilon_{c}=5.0 \mathrm{keV}\right)$ with up to three beamlines per wiggler offering a flux in excess of $4 \cdot 10^{13}$ at $12 \mathrm{keV}$ and two straight sections of undulators to provide high brilliance photon beams in the energy range from a few tens of eV to $2 \mathrm{keV}$. The resulting increase of storage ring circumference from $62.4 \mathrm{~m}$ to $100.8 \mathrm{~m}$ creates enough space at the inside of the ring to accommodate the injector synchrotron and the microtron preaccelerator. Figure 1 shows a foot print of the new storage ring lay-out.

\section{THE OPTICAL LATTICE}

The new optics is based on a 6 cell double bend achromat lattice with alternating high and low beta functions in the straights. For flexible matching of the straight sections a symmetric doublet structure is used in the high $\beta$ straights, and a symmetric quadruplet structure in the low $\beta$ sections, giving a total of 19 quadrupole families. The lattice functions of the unperturbed optics (no wigglers or undulators) are shown in Fig. 2 and the essential optical parameters are listed in Tab. 1. In the wiggler section a low vertical 


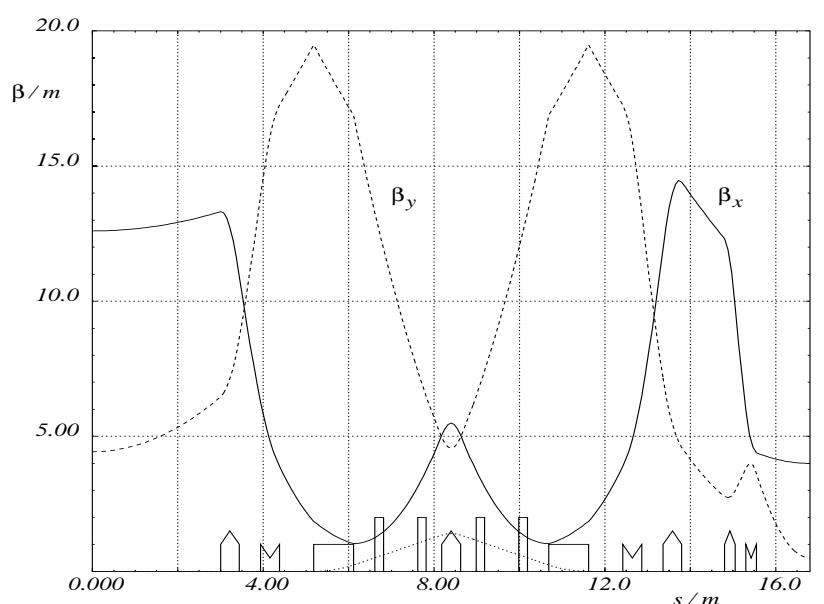

Figure 2: Optical functions of the unperturbed lattice for one sixth of the ring.

beta function is required to reduce the linear and nonlinear focusing effects of the wiggler. The simulation of the wiggler related effects are based on the assumption that there are only longitudinal and vertical field components, and no focusing or defocusing terms influencing the horizontal beam motion. The vertical beta function should not

Table 1: Storage ring parameters

\begin{tabular}{|l|c|}
\hline electron energy & $1.0 \mathrm{GeV}$ \\
beam current & $700 \mathrm{~mA}$ \\
circumference & $100.8 \mathrm{~m}$ \\
periodicity & 2.3 \\
working point $Q_{x} / Q_{y}$ & $5.2 / 3.2$ \\
nat. chromaticity $\xi_{x} / \xi_{y}$ & $-8.1 /-7.2$ \\
momentum compaction factor $\alpha$ & 0.005 \\
free space in high $\beta$ straight & $6 \mathrm{~m}$ \\
free space in low $\beta$ straight & $2.5 \mathrm{~m}$ \\
beta functions at the center of: & \\
$\quad$ high $\beta$-straight $\beta_{x} / \beta_{y}$ & $13 \mathrm{~m} / 4.5 \mathrm{~m}$ \\
$\quad$ low $\beta$ straight $\beta_{x} / \beta_{y}$ & $4 \mathrm{~m} / 0.5 \mathrm{~m}$ \\
max. beta function $\beta_{x} / \beta_{y}$ & $14.5 \mathrm{~m} / 19.5 \mathrm{~m}$ \\
max. dispersion $\eta$ & $1.4 \mathrm{~m}$ \\
\hline
\end{tabular}

differ too much from the rms bending radius ( $\rho=0.63 \mathrm{~m}$ at $1.0 \mathrm{GeV}$ ) of the electrons in the wiggler field. Tracking calculations indicated that there is a broad optimum for the dynamic aperture around $\beta_{y} \approx 0.5 \mathrm{~m}$. To get a high photon flux density from the wiggler beamlines the horizontal beta function in the wiggler section should be also small. From beam optical considerations a horizontal tune of $Q_{x}=5.2$ was chosen which gives a good behaviour of the optics together with a horizontal beta function of $\beta_{x} \approx 4 \mathrm{~m}$. The dynamic aperture of the bare lattice (Fig. 3) shows very good results, harmonic sextupoles are not required. The calculation is based on 1000 stable particle turns with small field and alignment errors assumed for the magnets, leading to $r m s$ closed orbit errors of about

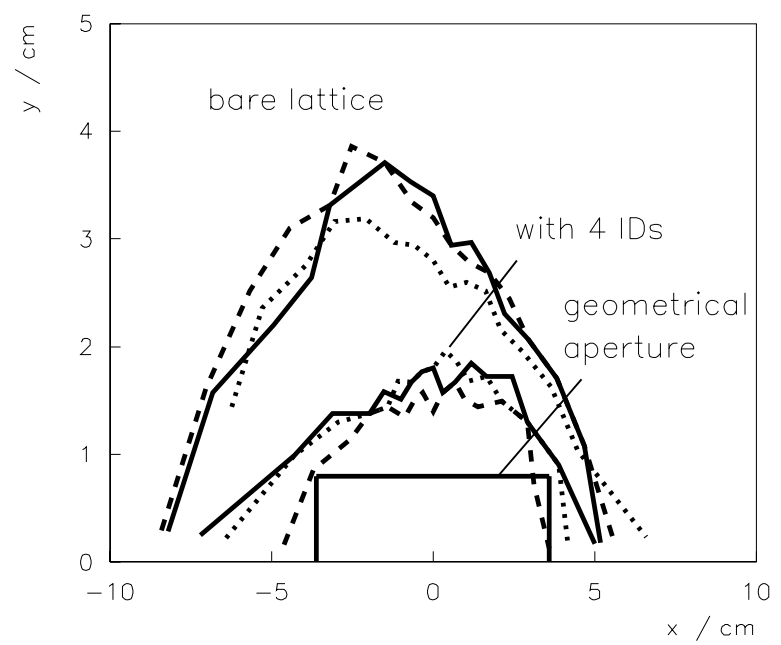

Figure 3: Dynamical and geometrical aperture in the middle of the high $\beta$ straight section for $\Delta p / p=0, \pm 2 \%$.

$1 \mathrm{~mm}$. The lattice has a large chromatic acceptance which is required for a good Touschek lifetime. The nonlinear chromatic tuneshift is smaller than 0.06 in the range of $-1.5 \% \leq \Delta p / p \leq+1.5 \%$, if the chromaticity is adjusted to $\Delta Q / \Delta p / p_{0}=+1$ in both planes. The dynamic aperture including two superconducting wigglers and two U49 undulators is shown in Fig. 3. Here the wigglers have been modeled by a 2-D scalar potential function starting from the wiggler field $B_{y}(s)$. As expected, the dynamic aperture is significantly reduced due to the nonlinearities of the insertion devices. With $\pm 30 \mathrm{~mm}$ horizontally and $\pm 15 \mathrm{~mm}$ vertically the dynamic aperture, however, is still comfortably large to guarantee stable beam operation.

The two $7.5 \mathrm{~T}$ wigglers enhance the radiation damping strongly and consequently reduce the equilibrium emittance significantly, a welcome feature of this source concept. A comparison of the transverse emittances, energy spread, energy loss per turn and related damping times for the bare lattice and with one to two wigglers are summarized in Tab. 2.

Table 2: 7.5 T Wiggler dependent damping effects

\begin{tabular}{|l|c|c|c|}
\hline bare lattice and $\mathrm{N}$ wigglers & $\mathrm{N}=0$ & $\mathrm{~N}=1$ & $\mathrm{~N}=2$ \\
\hline nat. emittance $\epsilon_{n}$ in $10^{-9} \mathrm{radm}$ & 115 & 70 & 50 \\
nat. energy width $\sigma_{e} / E_{0}$ in $10^{-4}$ & 6.5 & 8.0 & 9.0 \\
damping times $\tau_{x}=\tau_{y} / \tau_{s}$ in $\mathrm{ms}$ & $14 / 7$ & $8 / 4$ & $6 / 3$ \\
energy loss per turn in $\mathrm{keV}$ & 50 & 85 & 120 \\
\hline
\end{tabular}

\section{BEAM LIFETIME}

To estimate the lifetime at $1.0 \mathrm{GeV}$ for beam currents up to $700 \mathrm{~mA}$ gas scattering (elastic Coulomb and inelastic Bremsstrahlung scattering) and Touschek scattering has been considered. With a vertical acceptance of $A_{y}=11.6 \mathrm{~mm}$ mrad limited in the dipoles, a mean vertical beta function $\left\langle\beta_{y}\right\rangle=12.4 \mathrm{~m}$, a base pressure of 
$p_{0}=1 \cdot 10^{-9}$ mbar assuming a nitrogen $\left(\mathrm{N}_{2}\right)$-equivalent gas distribution and a pressure rise of $1.4 \cdot 10^{-10} \mathrm{mbar}$ per $100 \mathrm{~mA}$ of stored current, a total gas scattering lifetime of $8.7 \mathrm{~h}$ can be expected. The Touschek effect depends sensitively on the bunch volume, on the number of electrons per bunch and on the energy acceptance. Taking turbulent bunch lengthening with a longitudinal broadband impedance $|Z / n|$ of $3 \Omega$ and an energy acceptance of $\Delta E / E=1.3 \%$ into account for a bunch filling pattern where only $2 / 3$ of the 168 rf-buckets are filled the Touschek lifetime is $8.4 \mathrm{~h}$. As a result a total beam lifetime of about $4.2 \mathrm{~h}$ can be expected for a stored current of $700 \mathrm{~mA}$.

\section{THE 7.5 T MULTIPOLE WIGGLER}

The wigglers are based on the following concept [4]: A one meter long iron yoke has 15 poles (including 2 half poles at the ends) with a period length of $14 \mathrm{~cm}$ and a magnetic gap of $20 \mathrm{~mm}$. Each pole is excited by a racetrack shaped coil made from a suitable multifilament $\mathrm{NbTi}$ wire in a rectangular copper matrix. The coils have two separate windings to optimize the current with respect to the different critical field at the windings. Fig. 4 presents a vertical cut through the iron yoke showing the beam chamber with a $14 \mathrm{~mm}$ vertical aperture and the clamps for prestressing of the whole structure, and a horizontal cut showing the iron poles and the racetrack coils. The complete magnet assembly including the beam chamber is immersed in a liquid He bath.
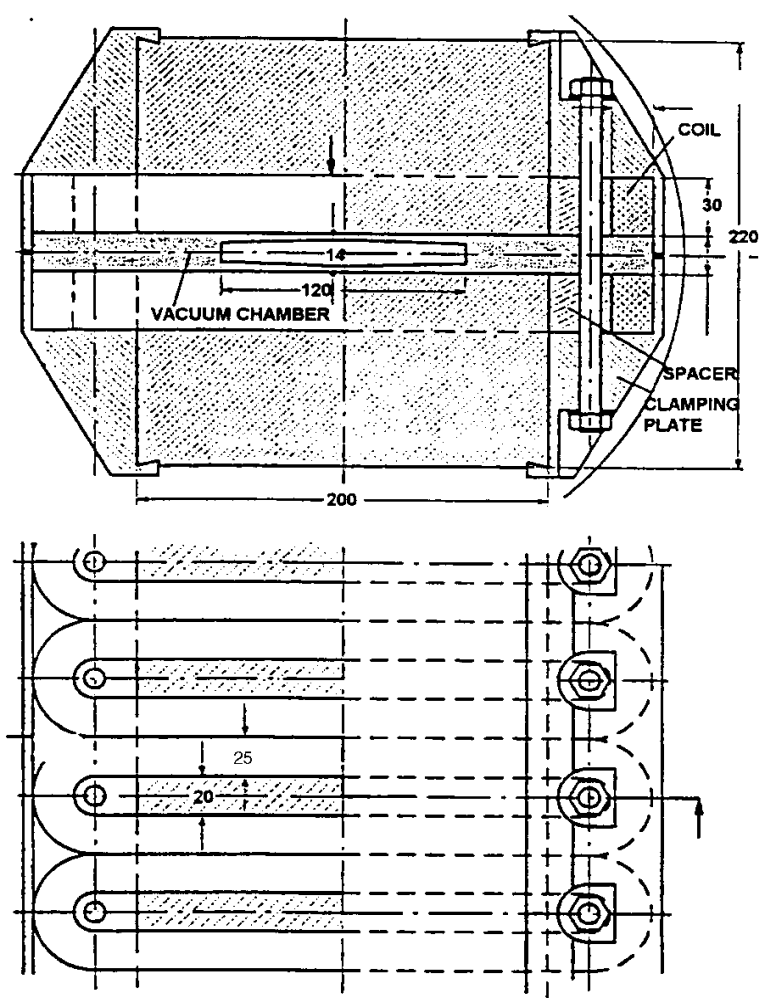

Figure 4: Vertical (top) and horizontal (bottom) cut through (a part of) the wiggler magnet.

\section{HARDWARE MODIFICATIONS}

Dipole magnets: To increase the energy from $800 \mathrm{MeV}$ to $1.0 \mathrm{GeV}$, the dipole field must be increased from $1.5 \mathrm{~T}$ to $1.875 \mathrm{~T}$. Reduction of the magnetic gap from $60 \mathrm{~mm}$ to $44 \mathrm{~mm}$ and narrowing the width of the pole from $190 \mathrm{~mm}$ to $90 \mathrm{~mm}$ allows to reach this relatively high field keeping the magnetic induction in the yoke at a tolerable level. Field calculations (2-D) indicate that after optimization of the pole profile the field homogeneity is better than $5 \cdot 10^{-4}$ within $\pm 15 \mathrm{~mm}$ horizontally. The dominant multipole is a sextupole which can be compensated in part by the chromatic sextupoles.

Vacuum system: A copper (CuSn2) vacuum chamber based on technology developed at DESY for HERA and DORIS is proposed which offers significant advantage in handling high radiation power densities. The dipole chamber can be fabricated by milling and vacuum brazing. Water cooling channels are brazed at the outside which can also be used for in-situ baking at temperatures up to $150^{\circ} \mathrm{C}$ by applying hot water at a pressure of 4.8 bar. A total of 48 ion getter pumps $(70 \mathrm{l} / \mathrm{s})$ supplemented by Ti sublimation pumps will be used to obtain a working pressure of $1 \cdot 10^{-9}$ mbar.

Injection system: The new topology with the synchrotron inside the storage ring favours horizontal injection, and with the straight section length of $6 \mathrm{~m}$ there is sufficient space available to place 4 kickers and a septum magnet in one high $\beta$ straight. With a max. strength of $8 \mathrm{mrad}$ a local injection bump can be produced with a horizontal amplitude of $20 \mathrm{~mm}$. A new transfer line must be built for beam transport from the synchrotron to the injection septum.

RF-system: Thanks to the higher beam energy and the two wigglers the radiated power is significantly enhanced as compared to BESSY I. For a beam current of $700 \mathrm{~mA}$ the power radiated in the dipoles and in the wigglers is $34.8 \mathrm{~kW}$ and $48.8 \mathrm{~kW}$ respectively. A minimum cavity voltage of $300 \mathrm{kV}$ must be provided for a $1.3 \%$ energy acceptance, giving a Touschek lifetime of $8 \mathrm{~h}$. With a second DORIStype cavity and two $70 \mathrm{~kW}$ rf-transmitters these conditions can be satisfied.

Beside the above modifications it is clear that after nearly 20 years of continuous operation some critical components and subsystems have to be replaced or refurbished. This is particularly true for the control system, the orbit measurement system and some of the power supplies.

\section{REFERENCES}

[1] G.v. Egan-Krieger, D. Einfeld, W.-D. Klotz, H. Lehr, R. Maier, G. Mülhaupt, R. Richter, E. Weihreter, IEEE-Trans. N.S., NS-30, No 4, 3103(1983)

[2] G.v. Egan-Krieger, D. Einfeld, H.-G. Hoberg, W.-D. Klotz, H. Lehr, R. Maier, M. Martin, G. Mülhaupt, R. Richter, L. Schulz, E. Weihreter, IEEE-Trans. N.S., NS-30, No 4, 3094(1983)

[3] BESSY Ia, Conceptual design study, BESSY, 1999

[4] G.-A. Voss, Internal Technical Note M99-01, DESY, 1999. 